\title{
Representations of $U_{\epsilon}^{r e s}\left(s l_{2}\right)$ via Restricted q-Fock Spaces
}

\author{
Xufeng Liu ${ }^{a}$ and Changpu Sun ${ }^{b}$ \\ ${ }^{a}$ Department of Mathematics, Peking University \\ Beijing 100871, P.R.China \\ ${ }^{b}$ Institute of Theoretical Physics, Chinese Academy of Sciences \\ Beijing 100080, China
}

\begin{abstract}
Two restricted $C\left[q, q^{-1}\right]$-forms of the well known q-boson algebra are introduced and the corresponding restricted q-Fock spaces defined. All of the irreducible highest weight representations, including the infinite dimensional ones, of $U_{\epsilon}^{\text {res }}\left(s l_{2}\right)$ of type 1 are constructed through the restricted q-Fock spaces.
\end{abstract}




\section{Introduction and Notations}

In this paper we take $q$ and $q^{-1}$ to be two indeterminates. We denote by $C(q)$ the field of rational functions of the indeterminate $q$ and denote by $C\left[q, q^{-1}\right]$ the ring of Laurent polynomials in the indeterminates $q$ and $q^{-1}$. As usual,the integer and nonnegative integer sets are denoted by $Z$ and $Z^{+}$respectively, while the positive integer set, or the natural number set, is denoted by $N$. For $n \in Z, m \in N$ we use the following notations

$$
\begin{aligned}
{[n]_{q} } & =\frac{q^{n}-q^{-n}}{q-q^{-1}},[m]_{q} !=[m]_{q}[m-1]_{q} \cdots[1]_{q} \\
{\left[\begin{array}{c}
n \\
m
\end{array}\right]_{q} } & =\frac{[n]_{q}[n-1]_{q} \cdots[n-m+1]_{q}}{[m]_{q} !}
\end{aligned}
$$

For $m \in Z \backslash N$ We adopt the convention

$$
\left[\begin{array}{c}
n \\
m
\end{array}\right]_{q}=0
$$

For $\varepsilon \in C,\left[\begin{array}{c}n \\ m\end{array}\right]_{\varepsilon}$ denotes the complex number obtained from $\left[\begin{array}{c}n \\ m\end{array}\right]_{q}$ by substituting $q=\varepsilon$ into the expression, as the symbol suggests.

Let $U_{q}$ be an associative algebra over $C(q)$. Naively, it is natural to think of $U_{q}$ as a family of algebras depending on a "parameter" q.Mathematically, this can be made precise as follows.If $\epsilon \in C$ is transcendental,one can specialize the indeterminate $q$ to $\epsilon$ by defining $U_{\epsilon}=U_{q} \otimes_{C(q)} C$, via the algebra homomorphism $C(q) \longrightarrow C$ that takes $q$ to $\epsilon$. When $\epsilon$ is algebraic the above homomorphism from $C(q)$ to $C$ is not available and this direct specialization of $U_{q}$ might not make sense. Nevertheless, one can proceed by first constructing a $C\left[q, q^{-1}\right]$-form , or integral form, of $U_{q}$, namely, a $C\left[q, q^{-1}\right]$-subalgebra $\tilde{U}_{q}$ of $U_{q}$ such that $U_{q}=\tilde{U}_{q} \otimes_{C\left[q, q^{-1}\right]} C(q)$. Then one defines the specialization $U_{\epsilon}$ of $U_{q}$ as $\tilde{U}_{q} \otimes_{C\left[q, q^{-1}\right]} C$, via the algebra homomorphism $C\left[q, q^{-1}\right] \longrightarrow C$ that takes $q$ to $\epsilon$.

The quantum algebra $U_{q}(g)$ associated to a Kac-Moody algebra $g$ is an associative algebra over $C(q)$. When $\epsilon$ is not a root of unity the representation theory of $U_{\epsilon}(g)$ has been well established[1]. To deal with the case where $\epsilon$ is a root of unity two $C\left[q, q^{-1}\right]$-forms of $U_{q}(g)$,namely,the "non-restricted" form and the "restricted" form, have been introduced.In the "non-restricted" form,one takes $\tilde{U}_{q}(g)$ to be the $C\left[q, q^{-1}\right]$-subalgebra of $U_{q}(g)$ generated by the Chevalley generators $e_{i}, f_{i}$ and some other elements of $U_{q}(g)$.In this case,the finite dimensional representations of $U_{\epsilon}(g)$ have been studied by De Concini,Kac and Procesi,when $g$ is finite dimensional,and by Beck and Kac,when $g$ is untwisted affine[2,3]. The restricted $C\left[q, q^{-1}\right]$-form $U_{q}^{r e s}(g)$ of $U_{q}(g)$ is introduced by Lusztig [4]. The study of representation theory of $U_{\epsilon}^{r e s}(g)$ is pioneered also by Lusztig and developed by Chari and Pressley $[5,6,7]$.

The q-boson realization method has been widely used to construct representations of quantum algebras in both generic case and root of unity case [8-16]. Especially, cyclic q-boson algebra has been introduced to obtain the so called cyclic representations of $U_{\epsilon}(g)$ 
associated with the non-restricted $C\left[q, q^{-1}\right]$-form of $U_{q}(g)[15,16]$. In this paper, we will introduce two restricted $C\left[q, q^{-1}\right]$-forms of the well known q-boson algebra and define the restricted q-Fock spaces correspondingly. Then we will construct all irreducible highest weight representations of $U_{\epsilon}\left(s l_{2}\right)$ of type 1 on the restricted q-Fock spaces.

\section{Some Basic Facts about $U_{\epsilon}^{r e s}\left(s l_{2}\right)$}

Let us recall some definitions and basic facts. For details we refer readers to Ref.[1].Throughout this paper we use the notation $\mathcal{A}=C\left[q, q^{-1}\right]$.

Definition 2.1. The quantum algebra $U_{q}\left(s l_{2}\right)$ is the associative algebra over $C(q)$ with generators $e, f, K$ and $K^{-1}$ and the following relations:

$$
\begin{aligned}
& K K^{-1}=K^{-1} K=1, \\
& K e K^{-1}=q^{2} e, K f K^{-1}=q^{-2} f, \\
& {[e, f]=\frac{K-K^{-1}}{q-q^{-1}} .}
\end{aligned}
$$

Definition 2.2. The algebra $U_{\mathcal{A}}^{r e s}\left(s l_{2}\right)$ is the $\mathcal{A}$-subalgebra of $U_{q}\left(s l_{2}\right)$ generated by the elements $e^{(r)}, f^{(r)}, K^{ \pm 1}(r \in N)$ for $r \geq 1$, where $e^{(r)}=\frac{e^{r}}{[r]_{q} !}$ and $f^{(r)}=\frac{f^{r}}{[r]_{q} !}$.

From now on we assume that $\epsilon$ is a primitive $p$ th root of unity, where $p$ is odd and greater than 1 . When necessary, $C$ is considered as $\mathcal{A}$-module via the the algebra homomorphism $\mathcal{A} \longrightarrow C$ that takes $q$ to $\epsilon$. By definition, the restricted specialization of $U_{q}\left(s l_{2}\right)$ is

$$
U_{\epsilon}^{r e s}\left(s l_{2}\right)=U_{\mathcal{A}}^{r e s}\left(s l_{2}\right) \otimes_{\mathcal{A}} C .
$$

For simplicity, $e^{(r)} \otimes_{\mathcal{A}} 1, f^{(r)} \otimes_{\mathcal{A}} 1$ and $K^{ \pm 1} \otimes_{\mathcal{A}} 1$ will be identified with $e^{(r)}, f^{(r)}$ and $K^{ \pm 1}$ respectively.

If $V$ is a representation of $U_{\epsilon}^{r e s}\left(s l_{2}\right)$ on which $K$ is diagonalizable and $K^{ \pm p}=1$ it is said to be of type 1 .

Definition 2.3. Let $m$ be an integer. The weight space $V_{m}$ of a $U_{\epsilon}^{r e s}\left(s l_{2}\right)$-module $V$ of type 1 is defined by

$$
V_{m}=\left\{v \in V \mid K v=\epsilon^{m} v,\left[\begin{array}{c}
K ; 0 \\
p
\end{array}\right]_{q} v=\left[\begin{array}{c}
m \\
p
\end{array}\right]_{\epsilon} v\right\}
$$

where

$$
\left[\begin{array}{c}
K ; 0 \\
p
\end{array}\right]_{q}=\prod_{s=1}^{p} \frac{K q^{1-s}-K^{-1} q^{s-1}}{q^{s}-q^{-s}}
$$

belongs to $U_{\mathcal{A}}^{r e s}\left(s l_{2}\right)$ and is identified with $\left[\begin{array}{c}K ; 0 \\ p\end{array}\right]_{q} \otimes_{\mathcal{A}} 1$.

For any integer $n$, write $n=n_{0}+p n_{1}$, where $n_{0}$ and $n_{1}$ are integers and $0 \leq n_{0}<p$. It is readily verified that

$$
\left[\begin{array}{l}
n \\
p
\end{array}\right]_{\epsilon}=n_{1}
$$


Then by definition, $v \in V$ is a weight vector of weight $m$ and we have

$$
K v=\epsilon^{m_{0}} v,\left[\begin{array}{c}
K ; 0 \\
p
\end{array}\right]_{q} v=m_{1} v .
$$

A $U_{\epsilon}^{r e s}\left(s l_{2}\right)$-module $V$ of type 1 is called a highest weight module if it is generated by a primitive vector, i.e. a vector $v_{\lambda}$ for some $\lambda \in Z$ such that $e v_{\lambda}=e^{(p)} v_{\lambda}=0$. It is obvious that for such a module $V$ we have

$$
V=\sum_{\mu \leq \lambda} \oplus V_{\mu}
$$

so that $\lambda$ is the highest weight of $V$ and $V \lambda_{\lambda}=C v_{\lambda}$. It then follows by the usual argument that $V$ has a unique irreducible quotient module.

Denote by $V_{\epsilon}^{\text {res }}(\lambda)$ the irreducible highest weight $U_{\epsilon}^{\text {res }}\left(s l_{2}\right)$-module of type 1 and of highest weight $\lambda$. Then by the Verma module construction and the above argument one can prove that $V_{\epsilon}^{\text {res }}(\lambda)$ is isomorphic to $V_{\epsilon}^{\text {res }}(\mu)$ if $\lambda=\mu$.

\section{Restricted q-Fock Spaces}

The $q$-boson algebra $B_{q}(n)$ of rank $n$ is the associative algebra over $C(q)$ generated by the elements $a_{i}, a_{i}^{+}, K_{i}^{ \pm 1}, 1(i=1,2, \cdots, n)$ with the following relations

$$
\begin{aligned}
a_{i} a_{i}^{+}-q^{2} a_{i}^{+} a_{i} & =1,\left[a_{i}, a_{j}^{+}\right]=0(i \neq j) \\
{\left[a_{i}, a_{j}\right] } & =\left[a_{i}^{+}, a_{j}^{+}\right]=0 \\
K_{i} a_{j}^{+} K_{i}^{-1} & =q^{\delta_{i j}} a_{j}^{+}, K_{i} a_{j} K_{i}^{-1}=q^{-\delta_{i j}} a_{j} \\
K_{i} K_{i}^{-1} & =K_{i}^{-1} K_{i}=1
\end{aligned}
$$

where 1 is the unit.

We introduce the notations

$$
a_{i}^{(n)}=\frac{a_{i}^{n}}{[n]_{q} !}, a_{i}^{+(n)}=\frac{a_{i}^{+n}}{[n]_{q} !}
$$

and the following definition.

Definition 3.1. The type 1 restricted $q-$ Boson algebra $B_{q}^{\text {res } 1}(2)$ of rank 2 is the $\mathcal{A}$-subalgebra of $B_{q}(2)$ generated by the elements $a_{i}, a_{i}^{+(r)}, K_{i}^{ \pm 1}, 1(i=1,2 ; r \in N)$; The type 2 restricted $q-$ Boson algebra $B_{q}^{\text {res } 2}(2)$ of rank 2 is the $\mathcal{A}$-subalgebra of $B_{q}(2)$ generated by the elements $a_{1}, a_{1}^{+(r)}, a_{2}^{(r)}, a_{2}^{+}, K_{1}^{ \pm 1}, K_{2}^{ \pm 1}, 1(r \in N)$.

By induction one can prove the following three lemmas without difficulty.

Lemma 3.1.

$$
\left[\begin{array}{l}
r \\
k
\end{array}\right]_{q}=q^{-k}\left[\begin{array}{c}
r-1 \\
k
\end{array}\right]_{q}+q^{r-k}\left[\begin{array}{l}
r-1 \\
k-1
\end{array}\right]_{q} \text { for } r \geq k \geq 0
$$




\section{Lemma 3.2.}

$$
\left[\begin{array}{l}
r \\
k
\end{array}\right]_{q} \in C\left[q, q^{-1}\right] \text { for } r \geq k \geq 0
$$

\section{Lemma 3.3.}

$$
\begin{aligned}
& a_{i}^{n} a_{i}^{+(m)}=\sum_{s=0}^{m} q^{n(s+m)} q^{\frac{(s-m)(s+m+1)}{2}}\left[\begin{array}{c}
n \\
m-s
\end{array}\right]_{q} a^{+(s)} a^{n-m+s} \\
& a_{i}^{(n)} a_{i}^{+m}=\sum_{s=0}^{m} q^{n(s+m)} q^{\frac{(s-m)(s+m+1)}{2}}\left[\begin{array}{c}
m \\
s
\end{array}\right]_{q} a^{+s} a^{(n-m+s)}
\end{aligned}
$$

Proposition 3.1. $\left\{a_{1}^{+\left(r_{1}\right)} a_{2}^{+\left(r_{2}\right)} a_{1}^{s_{1}} a_{2}^{s_{2}} K_{1}^{t_{1}} K_{2}^{t_{2}} \mid r_{i}, s_{i} \in Z^{+}, t_{i} \in Z ; i=1,2\right\}$ is an $\mathcal{A}$-basis of $B_{q}^{\text {res } 1}(2) ;\left\{a_{1}^{+\left(r_{1}\right)} a_{2}^{+r_{2}} a_{1}^{s_{1}} a_{2}^{\left(s_{2}\right)} K_{1}^{t_{1}} K_{2}^{t_{2}} \mid r_{i}, s_{i} \in Z^{+}, t_{i} \in Z ; i=1,2\right\}$ is an $\mathcal{A}$-basis of $B_{q}^{r e s 2}(2)$.

Proof. It follows directly from Lemma 3.2 and Lemma 3.3.

Corollary. $B_{q}^{\text {res } 1}(2)$ and $B_{q}^{\text {res } 2}(2)$ are two integral forms of $B_{q}(2)$.

Consider $B_{q}^{r e s 1}(2)$ as $B_{q}^{r e s 1}(2)$-module as well as $C\left[q, q^{-1}\right]$-algebra .Let $I$ be the left ideal of $B_{q}^{r e s 1}(2)$ generated by the elements

$$
K_{1}-1, K_{2}-1, a_{1}, a_{2} .
$$

Obviously, $I$ is $B_{q}^{\text {res } 1}(2)$-submodule. Let $\mathcal{F}_{\mathcal{A}}^{1}(2)$ denote the quotient module $B_{q}^{\text {res } 1}(2) / I$. It follows from Proposition 3.1 that $\mathcal{F}_{\mathcal{A}}^{1}(2)$ is a free $\mathcal{A}$-module and

$$
\left.\mathcal{F}_{\mathcal{A}}^{1}(2)=\operatorname{span}_{\mathcal{A}}\left\{a_{1}^{+\left(r_{1}\right)} a_{2}^{+\left(r_{2}\right)}|0\rangle\left|a_{1}\right| 0\right\rangle=a_{2}|0\rangle=0, K_{1}|0\rangle=K_{2}|0\rangle=|0\rangle ; r_{1}, r_{2} \in Z^{+}\right\} .
$$

where $\left\{a_{1}^{+\left(r_{1}\right)} a_{2}^{+\left(r_{2}\right)}|0\rangle \mid r_{1}, r_{2} \in Z^{+}\right\}$is a basis of $\mathcal{F}_{\mathcal{A}}^{1}(2) . \mathcal{F}_{\mathcal{A}}^{1}(2)$ is called $\mathcal{A}$-Fock module associated with $B_{q}^{\text {res } 1}(2)$. The $\mathcal{A}$-Fock module $\mathcal{F}_{\mathcal{A}}^{2}(2)$ associated with $B_{q}^{\text {res2 }}(2)$ can be defined in a similar way :

$\left.\mathcal{F}_{\mathcal{A}}^{2}(2)=\operatorname{span}_{\mathcal{A}}\left\{a_{1}^{+\left(r_{1}\right)} a_{2}^{+r_{2}}|0\rangle\left|a_{1}^{r}\right| 0\right\rangle=a_{2}^{(r)}|0\rangle=0, r \in N ; K_{1}|0\rangle=K_{2}|0\rangle=|0\rangle ; r_{1}, r_{2} \in Z^{+}\right\}$.

It is easy to see that $\mathcal{F}_{\mathcal{A}}^{1}(2) \otimes_{\mathcal{A}} C(q)=\mathcal{F}_{\mathcal{A}}^{2}(2) \otimes_{\mathcal{A}} C(q) \triangleq \mathcal{F}_{q}(2)$ is the ordinary q-Fock space associated with $B_{q}(2)$. It is a vector space over the field $C(q)$. If we regard $\mathcal{F}_{q}(2)$ as $\mathcal{A}$-module,then by identifying $\mathcal{F}_{\mathcal{A}}^{1}(2)$ with $\mathcal{F}_{\mathcal{A}}^{1}(2) \otimes_{\mathcal{A}} 1, \mathcal{F}_{\mathcal{A}}^{1}(2)$ becomes a submodule of $\mathcal{F}_{q}(2)$, and $\mathcal{F}_{\mathcal{A}}^{2}(2)$ becomes a submodule of $\mathcal{F}_{\mathcal{A}}^{2}(2)$ likewise. For convenience, in the subsequent discussion we will use the following notations:

$$
\begin{aligned}
& f\left(r_{1}, r_{2}\right)=a_{1}^{+\left(r_{1}\right)} a_{2}^{+\left(r_{2}\right)}|0\rangle, \\
& g\left(r_{1}, r_{2}\right)=a_{1}^{+\left(r_{1}\right)} a_{2}^{+r_{2}}|0\rangle .
\end{aligned}
$$

We define

$$
\begin{aligned}
& \mathcal{F}_{\epsilon}^{1}(2)=\mathcal{F}_{\mathcal{A}}^{1}(2) \otimes_{\mathcal{A}} C, \\
& \mathcal{F}_{\epsilon}^{2}(2)=\mathcal{F}_{\mathcal{A}}^{2}(2) \otimes_{\mathcal{A}} C,
\end{aligned}
$$

and identify $f\left(r_{1}, r_{2}\right) \otimes_{\mathcal{A}} 1$ and $g\left(r_{1}, r_{2}\right) \otimes_{\mathcal{A}} 1$ with $f\left(r_{1}, r_{2}\right)$ and $g\left(r_{1}, r_{2}\right)$ respectively. $\mathcal{F}_{\epsilon}^{1}(2)$ and $\mathcal{F}_{\epsilon}^{2}(2)$ will be referred to as restricted q-Fock spaces. In the following sections, we will construct irreducible representations of $U_{\epsilon}^{r e s}\left(s l_{2}\right)$ on the restricted q-Fock spaces. 


\section{Finite Dimensional Representations of $U_{\epsilon}^{r e s}\left(s l_{2}\right)$ via Restricted q-Fock Space}

We recall that $U_{q}\left(s l_{2}\right)$ has the following realization on $\mathcal{F}_{q}(2)$ [8] :

$$
\begin{aligned}
e & =K_{2}^{-1} a_{1}^{+} a_{2}, f=K_{1}^{-1} a_{1} a_{2}^{+}, \\
K & =K_{1} K_{2}^{-1}, K^{-1}=K_{1}^{-1} K_{2} .
\end{aligned}
$$

Remark. The above realization looks different from that presented in Ref.[8]. This is because the generators of the q-boson algebra adopted in this paper are slightly different from those in Ref.[8].

By the natural action $\mathcal{F}_{q}(2)$ becomes a $U_{q}\left(s l_{2}\right)$-module through this realization. Then naturally it becomes a $U_{\mathcal{A}}^{r e s}\left(s l_{2}\right)$-module through the realization

$$
e^{(r)}=q^{-\frac{r(r-1)}{2}} K_{2}^{-r} a_{1}^{+(r)} a_{2}^{r}, f^{(r)}=q^{-\frac{r(r-1)}{2}} K_{1}^{-r} a_{2}^{+(r)} a_{1}^{r} .
$$

It turns out that $\mathcal{F}_{\mathcal{A}}^{1}(2)$ is a $U_{\mathcal{A}}^{\text {res }}\left(s l_{2}\right)$-submodule. In fact, the action of $U_{\mathcal{A}}^{\text {res }}\left(s l_{2}\right)$ on $\mathcal{F}_{\mathcal{A}}^{1}(2)$ reads as follows:

$$
\begin{aligned}
K f\left(r_{1}, r_{2}\right) & =q^{r_{1}-r_{2}} f\left(r_{1}, r_{2}\right), K^{-1} f\left(r_{1}, r_{2}\right)=q^{r_{2}-r_{1}} f\left(r_{1}, r_{2}\right), \\
e^{(r)} f\left(r_{1}, r_{2}\right) & =\left[\begin{array}{c}
r+r_{1} \\
r
\end{array}\right]_{q} f\left(r_{1}+r, r_{2}-r\right), \\
f^{(r)} f\left(r_{1}, r_{2}\right) & =\left[\begin{array}{c}
r+r_{2} \\
r
\end{array}\right]_{q} f\left(r_{1}-r, r_{2}+r\right) .
\end{aligned}
$$

Here we have used Lemma 3.3 and the formula

$$
a_{i}^{+(m)} a_{i}^{+(n)}=\left[\begin{array}{c}
m+n \\
m
\end{array}\right]_{q} a_{i}^{+(m+n)} \text { for } i=1,2 ; m, n \in N .
$$

Thus it follows that $U_{\mathcal{A}}^{\text {res }}\left(s l_{2}\right) \mathcal{F}_{\mathcal{A}}^{1}(2) \subset \mathcal{F}_{\mathcal{A}}^{1}(2)$. This proves the claim.

Clearly, $\mathcal{F}_{\epsilon}^{1}(2)$ is a $U_{\mathcal{A}}^{\text {res }}\left(s l_{2}\right)$-module. It then follows that it is also a $U_{\epsilon}^{\text {res }}\left(s l_{2}\right)$-module. Here the action of $U_{\epsilon}^{r e s}\left(s l_{2}\right)$ on $\mathcal{F}_{\epsilon}^{1}(2)$ is induced from that of $U_{\mathcal{A}}^{r e s}\left(s l_{2}\right)$ by identifying $q$ with $\epsilon$. Explicitly, we have

$$
\begin{aligned}
K f\left(r_{1}, r_{2}\right) & =\epsilon^{r_{1}-r_{2}} f\left(r_{1}, r_{2}\right), K^{-1} f\left(r_{1}, r_{2}\right)=\epsilon^{r_{2}-r_{1}} f\left(r_{1}, r_{2}\right), \\
e^{(r)} f\left(r_{1}, r_{2}\right) & =\left[\begin{array}{c}
r+r_{1} \\
r
\end{array}\right]_{\epsilon} f\left(r_{1}+r, r_{2}-r\right), \\
f^{(r)} f\left(r_{1}, r_{2}\right) & =\left[\begin{array}{c}
r+r_{2} \\
r
\end{array}\right]_{\epsilon} f\left(r_{1}-r, r_{2}+r\right) .
\end{aligned}
$$

We observe that for $m \in N$ the subspace

$$
V_{m} \triangleq \operatorname{span}_{C}\left\{f\left(r_{1}, r_{2}\right) \mid r_{1}+r_{2}=m\right\}
$$


of $\mathcal{F}_{\epsilon}^{1}(2)$ is a $U_{\epsilon}^{\text {res }}\left(s l_{2}\right)$-submodule.Define $v_{i}^{(m)}=f(m-i, i)$ for $i=0,1, \cdots, m$. Then $\left\{v_{0}^{(m)}, \cdots, v_{m}^{(m)}\right\}$ is a basis of $V_{m}$.

The action of $U_{\epsilon}^{r e s}\left(s l_{2}\right)$ on $V_{m}$ is given by

$$
\begin{aligned}
K v_{r}^{(m)} & =\epsilon^{m-2 r} v_{r}^{(m)}, K^{-1} v_{r}^{(m)}=\epsilon^{2 r-m} v_{r}^{(m)}, \\
e v_{r}^{(m)} & =[m-r+1]_{\epsilon} v_{r-1}^{(m)}, f v_{r}^{(m)}=[r+1]_{\epsilon} v_{r+1}^{(m)}, \\
e^{(p)} v_{r}^{(m)} & =\left((m-r)_{1}+1\right) v_{r-p}^{(m)}, f^{(p)} v_{r}^{(m)}=\left(r_{1}+1\right) v_{r+p}^{(m)} .
\end{aligned}
$$

Here $v_{i}^{(m)}$ is understood as the zero vector if $i<0$ or $i>m$. We notice that $V_{m}$ is none other than the so called Weyl module $W_{\epsilon}^{r e s}(m)$ with maximal weight $m$. Consequently, we have the following result.

Proposition 4.1. $V_{m}$ is irreducible if and only if either $m<p$ or $m_{0}=p-1$; If $V_{m}$ is reducible it is not completely reducible.

A sketch of the proof of this proposition can be found in Ref.[1].The main point is the observation that

$$
V^{\prime} \triangleq \operatorname{span}_{C}\left\{v_{r}^{(m)} \mid m_{0}<r_{0}<p, r_{1}<m_{1}\right\}
$$

is the unique proper $U_{\epsilon}^{r e s}\left(s l_{2}\right)$-submodule of $V_{m}$.

It is an established fact that every finite dimensional irreducible $U_{\epsilon}^{r e s}\left(s l_{2}\right)$-module of type 1 is a quotient module of Weyl module. As we have realized the Weyl module $W_{\epsilon}^{r e s}(m)$ for an arbitrary $m \in N$ via the q-Fock space, we have actually presented qFock space construction for all finite dimensional irreducible representations of type 1of $U_{\epsilon}^{r e s}\left(s l_{2}\right)$.

\section{$5 \quad$ Infinite Dimensional Representations of $U_{\epsilon}^{r e s}\left(s l_{2}\right)$ via Restricted q-Fock Space}

In this section let us turn to consider q-Fock space construction for some infinite dimensional irreducible representations of $U_{\epsilon}^{r e s}\left(s l_{2}\right)$. To this end, we need to use another q-boson realization of $U_{q}\left(s l_{2}\right)$. Our starting point is the following well-known q-boson realization of $U_{q}\left(s l_{2}\right)$ on $\mathcal{F}_{q}(2)[13]$ :

$$
\begin{aligned}
K & =q^{-1} K_{1}^{-1} K_{2}^{-1}, K^{-1}=q K_{1} K_{2}, \\
e & =K_{1}^{-1} K_{2}^{-1} a_{1} a_{2}, f=-a_{1}^{+} a_{2}^{+} .
\end{aligned}
$$

By definition $\mathcal{F}_{q}(2)$ is a $U_{q}\left(s l_{2}\right)$-module, and thus a $U_{\mathcal{A}}^{\text {res }}\left(s l_{2}\right)$-module, the action on $\mathcal{F}_{q}(2)$ being the natural one.We have

$$
e^{(r)}=q^{-r(r-1)} K_{1}^{-r} K_{2}^{-r} a_{1}^{r} a_{2}^{(r)}, f^{(r)}=(-1)^{r} a_{1}^{+(r)} a_{2}^{+r} .
$$

Then it follows from Lemma 3.3 that

$$
\begin{aligned}
e^{(r)} g\left(r_{1}, r_{2}\right) & =\left[\begin{array}{c}
r_{2} \\
r_{2}-r
\end{array}\right]_{q} g\left(r_{1}-r, r_{2}-r\right), \\
f^{(r)} g\left(r_{1}, r_{2}\right) & =(-1)^{r}\left[\begin{array}{c}
r+r_{1} \\
r
\end{array}\right]_{q} g\left(r_{1}+r, r_{2}+r\right),
\end{aligned}
$$


namely, $U_{\mathcal{A}}^{\text {res }}\left(s l_{2}\right) \mathcal{F}_{\mathcal{A}}^{2}(2) \subset \mathcal{F}_{\mathcal{A}}^{2}(2)$. Consequently, $\mathcal{F}_{\mathcal{A}}^{2}(2)$, and hence $\mathcal{F}_{\epsilon}^{2}(2)$ is a $U_{\mathcal{A}}^{\text {res }}\left(s l_{2}\right)$-module. Finally, $\mathcal{F}_{\epsilon}^{2}(2)$ becomes a $U_{\epsilon}^{\text {res }}\left(s l_{2}\right)$-module in the obvious way. Explicitly, the action of $U_{\epsilon}^{r e s}\left(s l_{2}\right)$ on $\mathcal{F}_{\epsilon}^{2}(2)$ is as follows:

$$
\begin{aligned}
K g\left(r_{1}, r_{2}\right) & =\epsilon^{-\left(r_{1}+r_{2}+1\right)} g\left(r_{1}, r_{2}\right), K^{-1} g\left(r_{1}, r_{2}\right)=\epsilon^{\left(r_{1}+r_{2}+1\right)} g\left(r_{1}, r_{2}\right), \\
e^{(r)} g\left(r_{1}, r_{2}\right) & =\left[\begin{array}{c}
r_{2} \\
r_{2}-r
\end{array}\right]_{\epsilon} g\left(r_{1}-r, r_{2}-r\right), \\
f^{(r)} g\left(r_{1}, r_{2}\right) & =(-1)^{r}\left[\begin{array}{c}
r+r_{1} \\
r
\end{array}\right]_{\epsilon} g\left(r_{1}+r, r_{2}+r\right) .
\end{aligned}
$$

On $\mathcal{F}_{\epsilon}^{2}(2)$, we have

$$
\left[\begin{array}{c}
K ; 0 \\
p
\end{array}\right]_{q}=\prod_{s=1}^{p} \frac{K q^{1-s}-K^{-1} q^{s-1}}{q^{s}-q^{-s}}=\prod_{s=1}^{p} \frac{K_{1}^{-1} K_{2}^{-1} q^{-s}-K_{1} K_{2} q^{s}}{q^{s}-q^{-s}} .
$$

Thus

$$
\left[\begin{array}{c}
K ; 0 \\
p
\end{array}\right]_{q} g\left(r_{1}, r_{2}\right)=\left[\begin{array}{c}
-r_{1}-r_{2}-1 \\
p
\end{array}\right]_{\epsilon} g\left(r_{1}, r_{2}\right)=-\left(r_{1}+r_{2}+1\right)_{1} g\left(r_{1}, r_{2}\right)
$$

Hence, $g\left(r_{1}, r_{2}\right)$ is a weight vector of the weight $-\left(r_{1}+r_{2}+1\right)$ and $\mathcal{F}_{\epsilon}^{2}(2)$ is a $U_{\epsilon}^{r e s}\left(s l_{2}\right)$-module of type 1 .

For an arbitrary integer $s$, define

$$
V^{s}=\operatorname{span}_{C}\left\{g\left(r_{1}, r_{1}+s\right) \mid r_{1} \in Z^{+}\right\} .
$$

We observe that $V^{s}$ is a $U_{\epsilon}^{r e s}\left(s l_{2}\right)$-submodule and there is the following decomposition:

$$
\mathcal{F}_{\epsilon}^{2}(2)=\sum_{s \in Z} \oplus V^{s}
$$

For $s$ that satisfies $s_{0}=0$, namely, $s= \pm r p\left(r \in Z^{+}\right)$, we have the following result.

Proposition 5.1. For $r \in Z^{+}, V^{ \pm r p}$ is isomorphic to $V_{\epsilon}^{r e s}(-(r p+1))$.

Proof. We only need to consider the case of $V^{r p}$. The other case is similar. We first show that $V^{r p}$ is an infinite dimensional irreducible $U_{\epsilon}^{r e s}\left(s l_{2}\right)$-module.For $m \in Z^{+}$define $g(m)=g(m, m+r p)$. Then $\left\{g(m) \mid m \in Z^{+}\right\}$is a basis of $V^{r p}$. We have

$$
e g(m)=[m]_{\epsilon} g(m-1), f g(m)=-[m+1]_{q} g(m+1)
$$

and

$$
e^{(p)} g(n p)=n g((n-1) p), f^{(p)} g(n p-1)=(n-1) g((n+1) p-1)
$$

for $n \in N$. Using these relations, one can easily show that as $U_{\epsilon}^{r e s}\left(s l_{2}\right)$-module $V^{r p}$ can be generated by an arbitrary vector in it. The irreducibility is thus proved.

As shown above, $g(0)$ is a weight vector of the weight $-(1+r p)$. It is easy to see that it is actually a highest weight vector. On the other hand, it is clear that $V^{r p}$ is a highest weight module: $V^{r p}=U_{\epsilon}^{r e s}\left(s l_{2}\right) g(0)$. The claim then follows from the irreducibility of $V^{r p}$. 
Now let us study $V^{s}$ with $s_{0} \neq 0$. When $s>0$, define $v_{m}=g(m, m+s)$ and the subspace $V^{\prime}$ of $V^{s}$ :

$$
V^{\prime}=\operatorname{span}_{C}\left\{v_{m} \mid p>m_{0} \geq p-s_{0}\right\} .
$$

When $s<0$, we use $W^{s}$ to denote $V^{s}$ for convenience. Then

$$
W^{s}=\operatorname{span}_{C}\left\{g\left(r_{1}+|s|, r_{1}\right) \mid r_{1} \in Z^{+}\right\}
$$

We define $w_{m}=g(m+|s|, m)$ and the subspace $W^{\prime}$ of $W^{s}$ :

$$
W^{\prime}=\operatorname{span}_{C}\left\{w_{m} \mid 0 \leq m_{0}<p-s_{0}\right\} .
$$

It is not difficult to see that $V^{\prime}$ and $W^{\prime}$ are proper submodules of $V^{s}$ and $W^{s}$ respectively.

Proposition 5.2. $V^{\prime}$ is isomorphic to $V_{\epsilon}^{\text {res }}(\lambda)$ with $\lambda=-\left(p-s_{0}+\left(s_{1}+1\right) p+1\right)$.

Proof. By the explicit action of $U_{\epsilon}^{r e s}\left(s l_{2}\right)$ on $\mathcal{F}_{\epsilon}^{2}(2)$, it is not difficult to see that $V^{\prime}$ is irreducible and $V^{\prime}=U_{\epsilon}^{r e s}\left(s l_{2}\right) v_{p-s_{0}}$. Since $v_{p-s_{0}}$ is the highest weight vector of $V^{\prime}$ of the weight $-\left(p-s_{0}+\left(s_{1}+1\right) p+1\right)$, the proposition then follows.

Proposition 5.3. $V^{\prime}$ is the unique maximal proper $U_{\epsilon}^{r e s}\left(s l_{2}\right)$-submodule of $V^{s}$ with $s>0$ and $s_{0} \neq 0$.

Proof. Let $V^{\prime \prime}$ be an arbitrary proper $U_{\epsilon}^{r e s}\left(s l_{2}\right)$-submodule of $V^{s}$. To prove the proposition, we only need to show that it is included in $V^{\prime}$. We observe that $V^{\prime \prime}$ has the vector space decomposition

$$
V^{\prime \prime}=\sum_{\lambda \in \Lambda_{s}} \oplus\left(V_{\lambda}^{s} \bigcap V^{\prime \prime}\right)
$$

where $\Lambda_{s}$ stands for the weight set of $V^{s}$. Suppose $v$ is a vector in $V^{\prime \prime}$. Define

$$
\begin{aligned}
& \Lambda_{s}^{\prime}=\left\{\lambda \in \Lambda_{s} \mid \lambda_{0} \geq p-s_{0}\right\} \\
& \Lambda_{s}^{\prime \prime}=\left\{\lambda \in \Lambda_{s} \mid \lambda_{0}<p-s_{0}\right\}
\end{aligned}
$$

Then we can write $v=v_{1}+v_{2}$ where

$$
v_{1} \in \sum_{\lambda \in \Lambda_{s}^{\prime \prime}} \oplus V_{\lambda}^{s}, v_{2} \in \sum_{\lambda \in \Lambda_{s}^{\prime}} \oplus V_{\lambda}^{s} \in V^{\prime} .
$$

Now due to the above vector space decomposition of $V^{\prime \prime}$, we have $v_{1} \in V^{\prime \prime}$. On the other hand, if $v_{1}$ is a nonzero vector, it is not difficult to show that $U_{\epsilon}^{r e s}\left(s l_{2}\right) v_{1}=V^{s}$. Consequently, $v_{1}=0$ as $V^{\prime \prime}$ is a proper $U_{\epsilon}^{r e s}\left(s l_{2}\right)$-submodule by assumption. This means $v=v_{2} \in V^{\prime}$, namely, $V^{\prime \prime} \subset V^{\prime}$. The proposition is thus proved.

Corollary. For $V^{s}$ with $s>0$ and $s_{0} \neq 0, V^{s} / V^{\prime}$ is isomorphic to $V_{\epsilon}^{\text {res }}(-(s+1))$.

Proof. Since $V^{\prime}$ is a maximal proper submodule, $V^{s} / V^{\prime}$ is irreducible. Moreover, $V^{s} / V^{\prime}$ is a highest module with the highest vector $\bar{v}_{0} \triangleq v_{0}+V^{\prime}=g(0, s)+V^{\prime}$ of the weight $-(s+1)$. The corollary thus follows.

Remark. Obviously, the above studied $U_{\epsilon}^{r e s}\left(s l_{2}\right)$-modules $V^{n p}, V^{\prime}$ and $V^{s} / V^{\prime}$ are all infinite dimensional. We notice that they are isomorphic to the modules $V_{\epsilon}^{\text {res }}(\lambda)$ with $\lambda$ negative. This should be the case. Actually, if $\lambda$ is a positive integer $V_{\epsilon}^{\text {res }}(\lambda)$ must be finite dimensional. 
In the same way, we can prove the following result.

Proposition 5.4. $W^{\prime}$ is isomorphic to $V_{\epsilon}^{\text {res }}(-(|s|+1)) ; W^{s} / W^{\prime}$ is isomorphic to $V_{\epsilon}^{\text {res }}(\lambda)$ with $\lambda=-\left(p-|s|_{0}+\left(1+|s|_{1}\right) p+1\right)$.

In summary, we have constructed all the representations $V_{\epsilon}^{\text {res }}(\lambda)$ of $U_{\epsilon}^{\text {res }}\left(s l_{2}\right)$ with $\lambda \in Z$ via the restricted q-Fock spaces. According to the representation theory of $U_{\epsilon}^{\text {res }}\left(s l_{2}\right)$ they have exhausted all the irreducible highest representations of $U_{\epsilon}^{r e s}\left(s l_{2}\right)$ of type 1.Obviously, the method presented in this paper can readily be generalized to be applicable to the case of $U_{\epsilon}^{\text {res }}\left(s l_{n}\right)$. 


\section{References}

1. V.Chari and A.N.Pressley,A Guide to Quantum Groups,Cambridge University Press,Cambridge,1994

2. C.De Concini and V.G.Kac,Representations of quantum groups at roots of unity,in Operator Algebras, Unitary Representations,Enveloping Algebras and Invariant Theory,A.Connes,M.Euflo,A.Joseph and R.Rentschler (eds.),pp.471-506,Progress in Mathematics 92,Birkhauser,Boston,1990.

3. J.Beck and V.G.Kac,Finite dimensional representations of quantum affine algebras at roots of unity,J.Amer.Math.Soc.9(1996)391-423.

4. G.Lusztig, Quantum deformations of certain simple modules over enveloping algebras, Adv. Math. 70(1988)237-249.

5. G.Lusztig, Finite dimensional Hopf algebras arising from quantized universal enveloping algebras, J. Amer. Math. Soc. 3(1990)257-296.

6. G.Lusztig, Quantum groups at roots of 1, Geom. Dedicata 35(1990)89-113.

7. V.Chari and A.N.Pressley, Quantum affine algebras at roots of unity, Representation Theory 1(1997)280-328.

8. C.P.Sun and H.C.Fu, The q-deformed boson realization of the quantum group $S U_{q}(n)$ and its representations, J.Phys.A 22(1989)L983-986.

9. L.C.Biedenharn, The quantum group $S U_{q}(2)$ and a q-analogue of the boson operators, J.Phys. A 22(1989)L873-878.

10. A.J.Macfarlane, On q-analogue of the quantum harmonic oscillator and the quantum group $S U_{q}(2)$. J.Phys.A 22(1989)4581-4588.

11. C.P.Sun and M.L.Ge, The q-analogue of the boson algebra, its representation on the Fock space, and applications to the quantum group,J. Math. Phys. 32(1991)595598.

12. C.P.Sun and M.L.Ge, The q-deformed boson realization of representations of quantized universal enveloping algebras for q a root of unity: I.The case of $U_{q}(S L(l))$, J.Phys. A 24(1991)3265-3280.

13. C.P.Sun, X.F.Liu and M.L.Ge, A new q-deformed boson realization of quantum algebra $s l_{q}(2)$ and nongeneric $s l_{q}(2)$ R-matrices, J. Math. Phys. 32(1991)24092412 .

14. C.P.Sun and M.L.Ge, The q-deformed boson realization of representations of quantized universal enveloping algebras for q a root of unity: II. The subalgebra chain of $U_{q}\left(C_{l}\right)$, J.Phys. A 25(1992)401-410. 
15. C.P.Sun, X.F.Liu and M.L.Ge, Cyclic boson algebra and q-boson realization of cyclic representation of quantum algebra $s l_{q}(3)$ at $q^{p}=1$ J.Phys.A 25(1992)161-168.

16. C.P.Sun and M.L.Ge, Cyclic boson operators and new representations of $s l_{q}(2)$ at q a root of unity, J.Phys.A 24(1991)L969-973. 